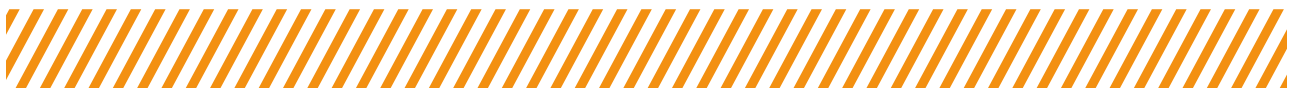

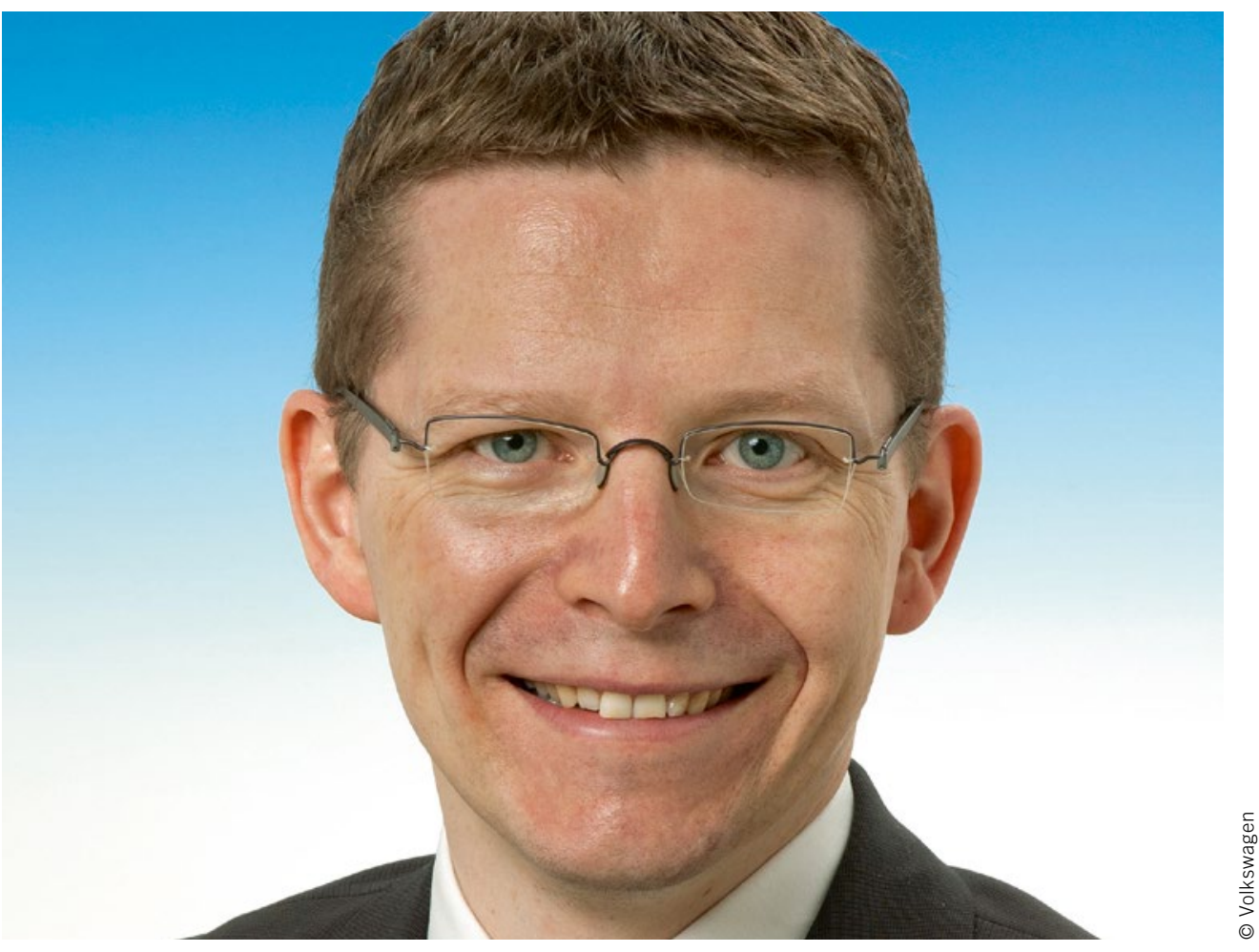

Dr. Gunnar Bärwaldt

Portfolio Manager Energy Services of the Brand Volkswagen and Leader of the VDA Working Group Net Integration

\section{Energy Revolution Needs Interpreters}

When people meet for the first time, they often mark the occasion by shaking hands. Subsequent meetings also typically begin and end with a handshake. However, if we fail to articulate our interests and concerns between handshakes in a way that others are able to comprehend, they will not be in a position to offer us an appropriate solution no matter how well-disposed they might be to doing so. This simple image underscores the importance and anticipates the complexity of finding a common language and a common set of terms in a new cross-sector environment that can be expected to play a crucial role in the effective digitization of our energy-supply systems.

What framework conditions have changed in the run-up to the energy transition? Our pursuit of a sustainable system of energy supply has led to the abandonment of nuclear power and the promotion of renewable sources of energy. This, in turn, has led to diminished flexibility on the supply side, a problem exacerbated by fluctuations relating to the integration of numerous small-scale production operations. It follows that the maintenance of a balanced system will depend on effective measures to enhance the fitness of the demand side for participation in a more sustainable, future-oriented system of energy supply. This will include tapping the potential available in the form of finely orchestrated approaches to consuming power and redistributing surplus power to the grid.
Who needs to communicate more effectively with whom? Photovoltaic systems, heat pumps and electric vehicles can be connected to energy-management systems that enable them to "talk" to energy suppliers and grid operators. When it comes to digitization, the "consumer" and the "prosumer" are essentially replaced by the "flexumer." However, individual flexumers can only be encouraged to play a role if they know what is expected of them - which they currently do not. Moreover, they tend to be equipped with devices that are made by different manufacturers and that use different communication standards and manufacturer-specific protocols.

What is the best approach to avoiding a Tower of Babel? The EEBus standard offers a kind of metalinguistic interface that enables flexumer devices to communicate with one another without first mastering any given number of other languages. The ISO 15118 charging standard can also be represented in EEBus. Vehicles and other integrated devices can thereby enhance the flexibility of flexumers and help them to meet their needs. By ensuring that all participants understand each other, the existing flexibilities can be used proactively. Situations that could lead to network overload can be avoided preventively.

We have the opportunity to join forces and make a valuable contribution to a digitalized and more sustainable energy sector. 